\title{
Cogeneration of algebras in regular categories
}

\section{Jiři Adámek}

\begin{abstract}
A quotient algebra of a given algebra is said to be cogenerated by a quotient ubject if it is contained in the quotient object and is the biggest with this property. Triples $T$ over a regular category are characterized which have the property that every quotient object of a T-algebra cogenerates some quotient algebra: these are precisely the right exact triples, preserving colimits of quotient chains. This improves a result of Michael Barr (J. Pure App 2. Algebra 4 (1974), 1-8) that every right exact, finitary triple has the investigated property. This result is related to categorical automata, since a triple has the above property iff triple machines admit a minimal realization of every behavior.
\end{abstract}

\section{Cogeneration and realization}

I.1. Cogeneration of quotient algebras is the dual notion to the generation of subalgebras. Given a triple $T$, a subobject of a T-algebra generates the least subalgebra, containing it. Dually, a regular quotient object cogenerates the biggest regular quotient algebra, contained in it. Here we use the current ordering of quotients of an object $A$ (that is, epis $e: A \rightarrow X)$, namely $e \leq e^{\prime}$ iff $e^{\prime}=k . e$ for some $k: X \rightarrow X^{\prime}$.

We work with a fixed triple $T=(T, \mu, n)$ over a category $K$; we say that $T$ has a certain property provided that $T$ has it.

I.2 DEFINITION. A triple $T$ is said to admit regular cogeneration

Received 29 June 1976. 
if for every T-algebra $(A, d)$ and every regular quotient $e$ of $A$ there exists the biggest regular quotient algebra $\varepsilon:(A, d) \rightarrow\left(A^{\prime}, d^{\prime}\right)$ with $\varepsilon \leq e$.

This notion is the algebraic form of minimal realizations of behaviors by T-machines; see [2, 4] or [5]. Roughly speaking, a T-machine is a T-algebra $(A, d)$ together with an "output" morphism $e: A \rightarrow X$. The quotient algebras, contained in $e$, present realizations (or reductions of the machine). The biggest quotient algebra yields the minimal realization (or reduction).

Let us remark here that Barr uses in [7] the dual order of quotients ( $e \leq e^{\prime}$ iff $e=k \cdot e^{\prime}$ ) which is more natural from the point of view of realizations. Yet, it seems to be a firm custom in algebra to use the order defined in I.I and we are going to follow this custom in the present paper.

I.3. Triples, which admit regular cogeneration, are characterized in $[2,4]$. Mild assumptions on the category $K$ are needed (for example, they are fulfilled by every cocomplete, co-well powered category in which every extreme epi is regular):

(i) $K$ has regular factorizations (that is, all regular epis and monics form a factorization system);

(ii) $K$ has big regular cointersections (that is, regular quotients of any object form a class-complete "lattice"; the infima are called cointersections).

THEOREM. Let $K$ be a category with properties (i) and (ii). Let $T$ be a regular triple over $K$ (that is, $T e$ is a regular epi as soon as $e$ is). Then $T$ admits regular cogeneration iff it preserves cointersections.

I.4. The above theorem is quite general, but in concrete situations it turns out to be difficult to decide whether a given functor preserves cointersections or not (see $[3,11]$ where this is solved for sets and vector spaces). The aim of the present paper is to show that for right exact triples (triples preserving coequalizers of equivalence relations) more handy conditions, concerning colimits of chains, are necessary and sufficient.

This paper uses a proof technique, developed by Barr $[6,7]$ and 
Grillet [8] for relations in a regular category.

\section{Strictly regular categories}

II.1. We recall from Grillet [8]:

DEFINITION. A category $K$ is regular if it has finite limits and regular factorizations and if pullbacks carry regular epis, that is, given a pullback $e . e^{\prime}=f . f^{\prime}$ where $e$ is a regular epi, so is $f^{\prime}$.

We need a stronger condition on $K$, concerning colimits of chains. Recall that, given a cardinal $\alpha$ (considered as the well-ordered category of all ordinals $i<\alpha$ ), a functor $X: \alpha \rightarrow K$ is called an $\alpha$-chain in $K$, or simply a chain. (If $\alpha=K_{0}$, we speak about sequences rather than chains.) We denote by $x_{i}$ the objects, and by $x_{i, j}: x_{i} \rightarrow x_{j}$ the morphisms $(i \leq j<\alpha)$.

If all $x_{i, j}$ are regular epis, we say that $X$ is a quotient chain. In this case, the colimit of $X$ is just the cointersection of $x_{0, i}$ $(i<\alpha)$; more precisely, if $v_{i}: X_{i} \rightarrow A \quad(i<\alpha)$ is the colimit of $X$ then $v_{0}$ is the cointersection of $x_{0, i}$. Conversely, every well-ordered cointersection is a colimit of a quotient chain.

II.2 DEFINITION. A regular category is strictly regular if it has finite colimits and colimits of chains, the latter commuting with pullbacks.

We shall show presently that a number of current categories are strictly regular. Yet, we also need a weaker condition. We use the phrase: colimits of sequences of split monics commute with pullbacks. This means that, given sequences $X, Y, Z$ of split monics and transformations $f: X+Z, g: Y \rightarrow Z$, the following holds. Let us form pullbacks

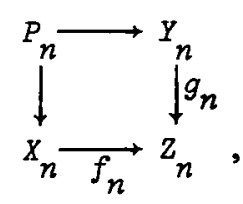

$n=0,1,2, \ldots$ (a new sequence $P$ arises, not necessarily of split 
monics, together with transformations $P \rightarrow X$ and $P \rightarrow Y$ ). Then $P$ has a colimit and the colimit-square

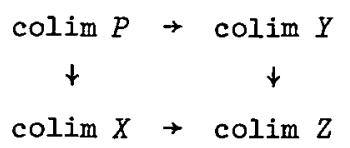

is a pullback, again.

DEFINITION. A regular category is S-regular if it has colimits of sequences of split monics and they commute with pullbacks.

EXAMPLE. Given a covariant set-functor $F$, denote by $S(F)$ the category of pairs $(X, R)$, where $X$ is a set and $R \subset F X$; morphisms from $(X, R)$ to $(Y, S)$ are mappings $f: X \rightarrow Y$ such that $F f$ sends $R$ into $S$; see [9]. It is rather easy to verify that the forgetful functor from $S(F)$ into sets creates limits and colimits and therefore $S(F)$ are strictly regular categories.

The categories of algebras of a given type, the category of graphs and, more generally, the categories of relational systems have the form $S(F)$; a big number of current categories are epi-reflective in some $S(F)$.

PROPOSITION. Let $K$ be a strictly regular category. Let $L$ be its full reflective subcategory, such that reflections are regular epis. If $L$ is closed to colimits of chains then it is strictly regular, too.

Proof. Standard reasoning shows that a given morphism in $L$ is a regular epi iff it is a regular epi in $K$. Since $L$ is closed to (finite) limits, it is regular. The strictness follows from the hypothesis that $L$ is closed to colimits of chains.

COROLLARY. Every variety of finitary algebras is a strictly regular category. Posets, tolerance spaces, partial algebras, and so on, form strictly regular categories.

PROPOSITION. Let $K$ be a strictly regular category and let $I$ be a small category. Then the functor-category $K^{I}$ is also strictly regular.

Proof. Regularity is (easily) proved in [8]. The strictness follows from the fact that both pullbacks and colimits of chains are computed "point-wise". 


\section{Relations}

III.1. Relations in a category are introduced and investigated by Grillet [8]. By a relation $R$ on an object $X$ is meant a subobject of $X \times X$ or, equivalently, a collectively monomorphic pair of morphisms $r^{\prime}, r^{\prime \prime}: R \rightarrow X$. The basic notion is the composition of relations. Given two relations $r^{\prime}, r^{\prime \prime}: R \rightarrow X$ and $s^{\prime}, s^{\prime \prime}: S \rightarrow X$, consider the pullback of $r^{\prime \prime}$ and $s^{\prime}$ :

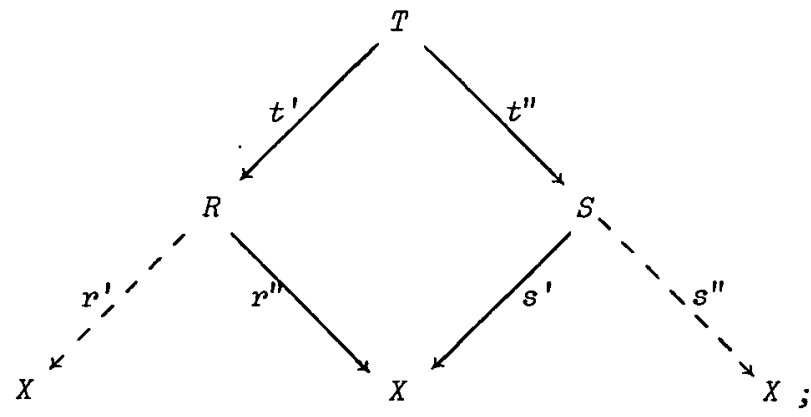

then the image of $\left(r^{\prime} . t^{\prime}\right) \times\left(s^{\prime \prime} . t^{\prime \prime}\right): T \times T \rightarrow X \times X$ is called the composition $R \circ S$. Here is where regularity comes in: the composition is associative in a regular category.

III.2. There are some obvious definitions. For example, a relation $R$ on $X$ is reflexive if it contains the diagonal $\Delta: X \rightarrow X \times X$; it is symmetric if $R^{-1} \subset R$ (where the inversion of a relation $r^{\prime}, r^{\prime \prime}$ is simply $r^{\prime \prime}, r^{\prime}$ ); and $R$ is transitive if $R \circ R \subset R$. A relation which has all these three properties is called an equivalence. Following Barr [7] we define:

DEFINITION. A functor from a regular category is pight exact if it preserves coequalizers of equivalence relations $R \rightarrow X$.

Right exactness is a comparatively mild condition - it says little more than the preservation of regular epis. For example, every functor from sets or from vector spaces is right exact.

Regular categories have kernel pairs; that is, for every morphism $f: X \rightarrow Y$ there exists a pair $e^{\prime}, e^{\prime \prime}: E \rightarrow X$ such that

(i) $f \cdot e^{\prime}=f \cdot e^{\prime \prime}$;

(ii) whenever $f \cdot k^{\prime}=f \cdot k^{\prime \prime}$ then there is a unique $r$ with 


$$
k^{\prime}=e^{\prime} \cdot r \text { and } k^{\prime \prime}=e^{\prime \prime} \cdot r
$$

It is easy to see that every kernel pair is an equivalence. Barr [7] assumes that, conversely, every equivalence is a kernel pair. Fortunately, we can do without this hypothesis; it excludes a number of important regular categories (for example, with.graphs, an equivalence $E \subset X \times X$ need not be a full subgraph of $X \times X$ - a regular monic - which is always the case for a kernel pair, of course).

III.3. Following Grillet, we "construct" the least equivalence, containing a given relation.

CONSTRUCTION. Let $R \stackrel{\rightarrow}{\rightarrow} X$ be a relation. Put $R^{\prime}=R \cup \Delta$ and define

$$
\begin{aligned}
S^{(1)} & =R^{\prime} \circ\left(R^{\prime}\right)^{-1}, \\
S^{(n+1)} & =S^{(n)} \circ S^{(1)} .
\end{aligned}
$$

Since $\Delta \subset s^{(1)}$ (and, moreover, this inclusion is a split monic), we have $S^{(n)}=S^{(n)} \circ \Delta \subset S^{(n+1)}$ and we get a sequence

$$
S^{(1)} \rightarrow S^{(2)} \rightarrow S^{(3)}
$$

of split monics. We denote by $R^{*} \stackrel{\rightarrow}{\rightarrow} X$ the union of this sequence:

$$
R^{*}=\bigcup_{n=1}^{\infty} S^{(n)} .
$$

THEOREM. Let $K$ be an S-regular category. Then for every relation $R \stackrel{\rightarrow}{\rightarrow}$,

(i) $R^{*}$ is the least equivalence, containing $R$;

(ii) the coequalizers of $R \rightarrow X$ and $R^{*} \rightarrow X$ are the same.

Proof. We denote the relation-maps by

$$
r^{\prime}, r^{\prime \prime}: R+X \text { and } s_{n}^{\prime}, s_{n}^{\prime \prime}: S^{(n)} \rightarrow X .
$$

(A) $R^{*}$ is an equivalence.

Since all $S^{(n)}$ are reflexive and symmetric, so is $R^{*}=U S^{(n)}$. Let us verify the transitivity. We have $s^{(n)} \circ S^{(n)}=S^{(2 n)}$, defined via the pullback 


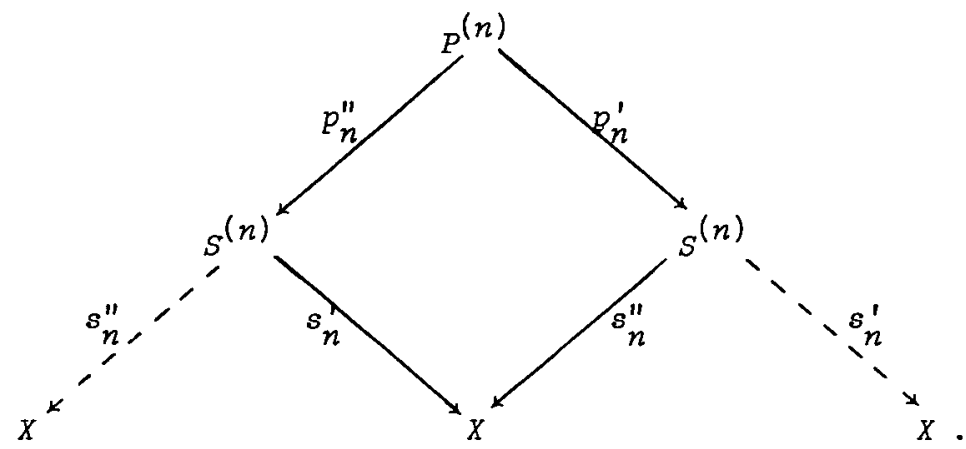

More in detail; $S^{(2 n)}$ is the image of $\left(s_{n}^{\prime} \cdot p_{n}^{\prime}\right) \times\left(s_{n}^{\prime \prime} \cdot p_{n}^{\prime \prime}\right)$ and, via $s^{(2 n)} \subset R^{*}$, we get $k_{n}: P^{(n)} \rightarrow R^{*}$ with $s_{n}^{\prime} \cdot p_{n}^{\prime}=r^{\prime} \cdot k_{n}$ and $s_{n}^{\prime \prime} \cdot p_{n}^{\prime \prime}=r^{\prime \prime} \cdot k_{n}$. Since $S^{(n)}$ is a sequence of split monics (while $X$ is a constant sequence), we can use the S-regularity: the colimit-square

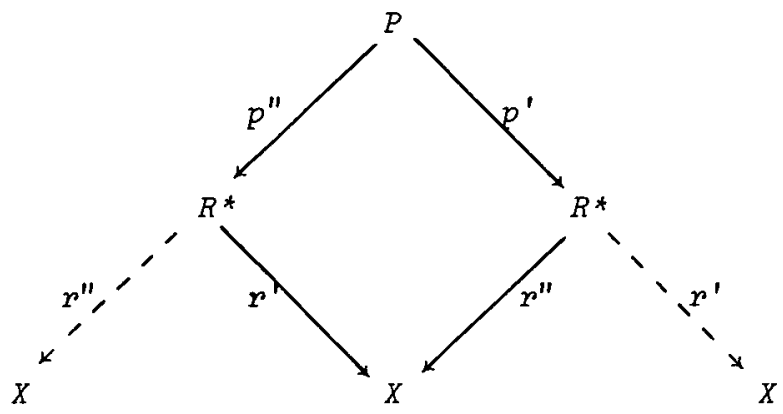

is a pullback, again. By the definition of composition of relations, $R^{*} \circ R^{*}$ is the image of $\left(r^{\prime} \cdot p^{\prime}\right) \times\left(r^{\prime \prime} \cdot p^{\prime \prime}\right)$. We have $k: P \rightarrow R^{*}$ as a colimit of the above $k_{n}$; since $\left(s_{n}^{\prime} \cdot p_{n}^{\prime}\right) \times\left(s_{n}^{\prime \prime} \cdot p_{n}^{\prime \prime}\right)=\left(p^{\prime} \times r^{\prime \prime}\right) \cdot k_{n}$, we get $\left(r^{\prime} \cdot p^{\prime}\right) \times\left(r^{\prime \prime} \cdot p^{\prime \prime}\right)=\left(r^{\prime} \times r^{\prime \prime}\right) \cdot k$. Thus $R^{*} \circ R^{*}$ is contained in $R^{*}$ (which equals the image of $r^{\prime} \times r^{\prime \prime}$ ).

(B) $R^{*}$ is the least equivalence.

Indeed, if $E$ is an equivalence, then $R \subset E$ implies $R^{\prime} \subset E$, hence $S^{(1)} \subset E \circ E^{-1} \subset E$ and, analogously, $S^{(n)} \subset E$. Therefore $R^{*} \subset E$. (C) Given $X \rightarrow Y$, then $R^{*} \stackrel{\rightarrow}{\rightarrow} X \rightarrow Y$ commutes iff $R \stackrel{\rightarrow}{\rightarrow} X \rightarrow Y$ does. The necessity follows from the fact that $R \subset R^{*}$. The sufficiency 
follows from the above: if $R \rightarrow X \rightarrow Y$ commutes, then $R$ is contained in the kernel pair of $X+Y$, which is an equivalence; thus $R^{*}$ is contained in it, too.

Now $(i i)$ follows.

III.4. The following theorem is essentially Theorem 1.3 of Barr [7]. Yet, we exhibit a full proof, because the theorem plays a central role in the present paper and our hypotheses are considerably weaker than Barr's. The corollary below is from the same paper, 1.5 .

A coequalizer $Y \stackrel{\rightarrow}{\rightarrow} X \rightarrow Z$ is reflexive if the image of $Y \stackrel{\rightarrow}{\rightarrow} X$ in $X \times X$ contains $\Delta$ (that is, it is a reflexive relation).

THEOREM. Let $K$ be an S-regular category and let $T$ be a right exact triple, preserving colimits of sequences of split monics. Then the forgetful functor from $T$-algebras, $U: K^{\top} \rightarrow K$, preserves reflexive coequalizers.

Proof. (A) $K^{\top}$ is an $S$-regular category.

It is evident that $U$ creates all existing limits and all those colimits which $T$ preserves. Thus, $U$ creates finite limits, coequalizers of equivalences (and equivalences, too) as well as colimits of sequences of split monics. Since every regular epi is the coequalizer of its kernel pair (which is an equivalence), $U$ creates regular epis. Therefore, $K^{\top}$ is $S$-regular.

(B) $U$ preserves the calculus of relations.

Since $U$ preserves monics and regular epis, it preserves factorizations, hence relations. It also preserves pullbacks, hence composition. Since $U$ also preserves colimits of sequences of split monics, it preserves the "star" from the preceding theorem for reflexive relations: $U\left(R^{*}\right)=(U R)^{*}$ if $\Delta \subset R$ (that is, if $R^{\prime}=R$ ).

(C) $U$ preserves reflexive coequalizers.

It is evident that a pair $Y \stackrel{\rightarrow}{\rightarrow} X$ has the same coequalizer as its image (in $X \times X$ ); since $U$ preserves images, it suffices to verify that $U$ preserves coequalizers of reflexive relations. Since $U$ preserves the "star" of these relations, it follows from (ii) in the preceding theorem, that the preservation of coequalizers of equivalences is sufficient. As 
noted above, $U$ preserves these coequalizers and the theorem follows.

COROLLARY. Under the scome hypotheses, $U$ preserves pushouts of regular epis (that is, finite cointersections).

\section{Properties of functors}

IV.1. In this section we show some interrelations between the preservation of colimits of mono-chains and epi-chains. This is an interesting example of the strength of the strict regularity; and the results obtained will be fundamental for the last section.

We recall that a limit is absolute if every functor preserves it.

IV.2 LEMMA. Let $X$ be a sequence of split monics with a colimit $v_{n}: X_{n} \rightarrow A$. Then also $v_{n}$ are split monics. Moreover, the pushouts

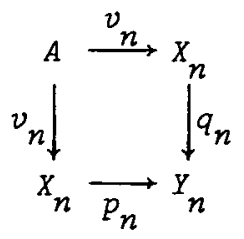

are absolute pullbacks.

Proof. Since $X_{n, n+1}$ are split monics, we have $k_{n}: X_{n+1} \rightarrow X_{n}$ with $k_{n} \cdot X_{n, n+1}=1$. For a given $n_{0}$ we can form the following compatible family $h_{n}: X_{n} \rightarrow X_{n_{0}}: h_{n_{0}}=1 ; h_{n}=X_{n, n_{0}}$ if $n<n_{0}$ and $h_{n}=k_{n_{0}} \cdot \ldots \cdot k_{n-1}$ if $n>n_{0}$. Then there exists $h: A \rightarrow x_{n_{0}}$ with $h \cdot v_{n}=h_{n}$. Thus $v_{n_{0}}$ splits, for $h \cdot v_{n_{0}}=1$.

The proposition about pushouts holds, more generally, for an arbitrary split monic: since $1 \cdot v_{n}=\left(v_{n} \cdot h\right) \cdot v_{n}$ (because $h \cdot v_{n}=1$ ), there exists $h^{\prime}: Y_{n} \rightarrow X_{n}$ such that $I=h^{\prime} \cdot p_{n}$ and $v_{n} \cdot h=h^{\prime} \cdot q_{n}$. Thus the above square is what could be called the split square. Standard reasoning shows that this is an absolute pullback.

THEOREM. Let $K$ be a strictly regular category. If a fronctor $F: K \rightarrow K$ preserves colimits of quotient sequences, then $F$ also 
preserves colimits of sequences of split monics.

Proof. Let $X$ be a sequence of split monics with a colimit $v_{n}: X_{n} \rightarrow A \cdot$ Let

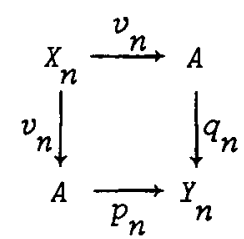

denote pushouts. Then for every $n$ clearly $q_{n+1} \cdot v_{n}=p_{n+1} \cdot v_{n}$ and we get a (unique) $Y_{n, n+1}: Y_{n} \rightarrow Y_{n+1}$ with

$$
y_{n, n+1} \cdot p_{n}=p_{n+1} \text { and } y_{n, n+1} \cdot q_{n}=q_{n+1} \text {. }
$$

Moreover, it is easy to verify that $y_{n, n+1}$ is the coequalizer of $p_{n} \cdot v_{n+1}$ and $q_{n} \cdot v_{n+1}$. In this way we obtain a quotient sequence $Y$.

Since $1 . v_{n}=1 . v_{n}$, we get a unique $w_{n}: Y_{n} \rightarrow A$ with

$w_{n} \cdot p_{n}=w_{n} \cdot q_{n}=1$. Let us verify that these morphisms $w_{n}$ form the colimit of $Y$. They are evidently compatible. Given another compatible family $u_{n}: y_{n} \rightarrow B$, we are to find $h: A \rightarrow B$ with $h . w_{n}=u_{n}(h$ is then unique, of course). We have a compatible family for $X$; $u_{n} \cdot p_{n} \cdot v_{n}: X_{n} \rightarrow B$ and so we get $k: A \rightarrow B$ with $k \cdot v_{n}=u_{n} \cdot p_{n} \cdot v_{n}$. For a fixed $n_{0}$ we have

$$
\left(u_{n_{0}} \cdot p_{n_{0}}\right) \cdot v_{n}=u_{n_{0}} \cdot y_{n_{0}}, n \cdot p_{n} \cdot v_{n}=u_{n} \cdot p_{n} \cdot v_{n}=k \cdot v_{n}
$$

for all $n \geq n_{0}$; therefore, $u_{n_{0}} \cdot p_{n_{0}}=k$ (for every $n_{0}$ ). Analogously $u_{n_{0}} \cdot q_{n_{0}}=k$. Therefore, for every $n$ we have

$$
\left(k \cdot w_{n}\right) \cdot p_{n}=u_{n} \cdot p_{n} \text { and }\left(k \cdot w_{n}\right) \cdot q_{n}=u_{n} \cdot q_{n}
$$

that is, $k \cdot w_{n}=u_{n}$. This is the morphism we want

By hypothesis, $F$ preserves this colimit; thus $F w_{n}$ present the 
colimit of FY. Furthermore, by the above lemma,

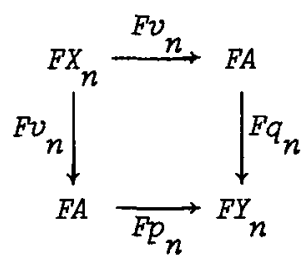

are pullbacks. Since $K$ is $S$-regular, the colimit square

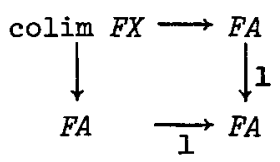

is a pullback. Thus $F A=\operatorname{colim} F X$ (together with the natural morphisms), which proves the theorem.

IV.3. If we want to generalize the above theorem to all chains of monics, we must "compensate" for the missing lemma. We shall assume about $K$ that

(i) it is strictly regular,

(ii) colimit injections of a chain of monics are monics,

(iii) given a monic $m$, the pushout (amalgam) of $m$ and $m$ is also a pullback.

The following theorem has an analogous proof to the one above.

THEOREM. Let $K$ fulfil (i)-(iii) above; let $F: K \rightarrow K$ be $a$ functor which preserves finite intersections and colimits of $\alpha$-chains of regular epis. Then $F$ preserves also colimits of $\alpha$-chains of monics.

IV.4. LEMMA. If $K$ is strictly regular, then the colimit of an increasing chain of equivalences is an equivalence. More in detail, given a chain of monics $E$ with a colimit $E^{*}$ and given compatible equivalences $E_{i} \stackrel{\rightarrow}{\rightarrow} X$, then the colimit pair $E^{*} \stackrel{\rightarrow}{\rightarrow} X$ is also an equivalence.

Proof. This is analogous to part (A) of the proof of Theorem III.3: here we consider the chain $E$ instead of the sequence $S^{(n)}$ of split monics; this is possible, since by hypothesis $K$ is strictly regular (not only S-regular). 
THEOREM. Let $K$ be a strictly regular category and let $F: K \rightarrow K$ be a right exact functor which preserves colimits of a-chains of monics. Then $F$ preserves also colimits of $\alpha$-chains of regular epis.

Proof. Let $X$ be an $\alpha$-chain of regular epis with a colimit $v_{i}: X_{i} \rightarrow A \quad(i<\alpha)$. Let $a_{i}, b_{i}: E_{i} \rightarrow X_{0}$ denote the kernel pair of $x_{0, i}$; then we get a natural chain $E$ of monics, defined by $a_{i}=a_{j} . E_{i, j}$ and $b_{i}=b_{j} E_{i, j}$ for $i \leq j<\alpha$. Let $E^{*}$ denote the colimit of $E$; then the colimit pair $a^{*}, b^{*}: E^{*} \rightarrow X_{0}$ is an equivalence by the above lemma. Let us verify that $v_{0}: X_{0} \rightarrow A$ is the coequalizer of $a^{*}, b^{*}$. Since for all $i$ we have

$$
v_{0} \cdot a_{i}=v_{i} \cdot X_{0, i} \cdot a_{i}=v_{i} \cdot X_{0, i} \cdot b_{i}=v_{0} \cdot b_{i},
$$

clearly $v_{0} \cdot a^{*}=v_{0} \cdot b^{*}$. Given another morphism $f: X_{0} \rightarrow A^{\prime}$ with $f \cdot a^{*}=f \cdot b^{*}$, we have $f \cdot a_{i}=f \cdot b_{i}$ for every $i$. Since $x_{0, i}$ is a regular epi, it is the coequalizer of its kernel pair $a_{i}, b_{i}$; therefore we get $v_{i}^{\prime}: X_{i}+A^{\prime}$ with $v_{i}^{\prime} \cdot X_{0, i}=f$. Then $\left\{v_{i}^{\prime}\right\}$ is a compatible family for $X$ and there is $g: A \rightarrow A^{\prime}$ with $f=g \cdot v_{0}$. Hence $v_{0}$ is the coequalizer of $a^{*}, b^{*}$.

Since $F$ is a right exact functor, $F v_{0}$ is the coequalizer of $F a^{*}, F b^{*}$. Now we are prepared to prove that the morphisms $\left\{F v_{i}\right\}$ constitute the colimit of $F X$. Let $\left\{w_{i}\right\}$ be an arbitrary compatible family for $F X, w_{i}: F X_{i} \rightarrow B$. For every $i$ we have

$$
w_{0} \cdot F a_{i}=w_{i} \cdot F X_{0, i} \cdot F a_{i}=w_{i} \cdot F X_{0, i} \cdot F b_{i}=w_{0} \cdot F b_{i}
$$

and, since $F$ preserves the colimit of $E$, there follows $w_{0} \cdot F a^{*}=w_{0} \cdot F b^{*}$. Therefore there exists $h: F A \rightarrow B$ with $w_{0}=h \cdot F v_{0}$; then, for every $i$,

$$
w_{i} \cdot F X_{0, i}=\left(h \cdot F v_{i}\right) \cdot F X_{0, i}
$$

thus $w_{i}=h \cdot F v_{i}$ for $F X_{0, i}$ is an epi $\left(X_{0, i}\right.$ is a regular epi and $F$ is right exact; hence it preserves regular epis). The uniqueness of $h$ is 
clear. The proof is concluded.

IV.5. The following lemma has been proved (implicitly but with all details) by Reiterman [10]. Colimits of chains are said to preserve a morphism class $M$ if, for every $M$-transformation between two chains, the natural map between their colimits is in $M$, too. For example, if these colimits commute with pullbacks, they preserve monomorphisms.

LEMMA. Let $K$ be a category with a factorization system ( $E, M)$. Let $K$ have colimits of $\alpha$-chains and let them preserve M-monics. Then every endofunctor, preserving colimits of $\alpha$-chains of both $M$-monics and E-epis, preserves colimits of all $\alpha$-chains.

Outline of the proof. Given an $\alpha$-chain $X$, consider the E-M-factorizations $X_{i} \rightarrow X_{j}^{(i)} \rightarrow X_{j}$ of the morphisms $x_{i, j}$. Via the diagonal fill-in, we obtain for each fixed $i$ a new $\alpha$-chain $x^{(i)}$ of E-epis:

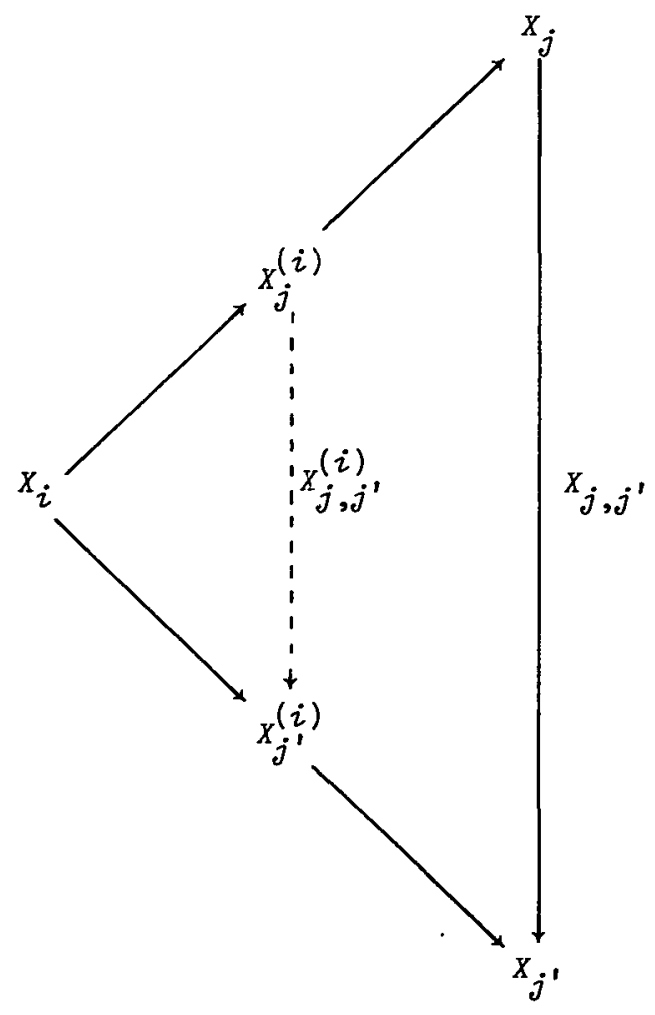


(putting $x_{j}^{(i)}=1$ for $j<i$ ).

Denote by $T_{i}$ the colimit of the chain $x^{(i)}$; recall that $F$ preserves this colimit. For $i \leq i^{\prime}$ we have a natural transformation $x^{(i)} \rightarrow x^{\left(i^{\prime}\right)}$ of M-monics. This yields a natural morphism $T_{i} \rightarrow T_{i}$, which, by hypothesis, also belongs to $M$. In this way we obtain an $\alpha$-chain $T$ of M-monics. Its colimit, again preserved by $F$, coincides with the colimit of $X$.

COROLLARY. Let $F$ be a right exact endofunctor of a strictly exact category. If $F$ preserves colimits of $\alpha$-chains of monics, then it preserves all colimits of a-chains.

\section{Main results}

V.1 THEOREM. Let $T$ be a regular triple over a strictly regular, co-well powered category. Then $T$ admits regular cogeneration if and only if it is right exact and preserves colimits of quotient chains.

Proof. (A) $K$ fulfils the hypotheses of Theorem I.3.

Since $K$ is strictly regular, it has regular factorizations as well as finite cointersections and colimits of quotient chains. There follows that $K$ has regular cointersections: since $K$ is co-well powered, regular quotients of an object form a (small) lattice, via the finite cointersections, in which every well-ordered subset has a supremum, via the colimits of chains. Consequently, this lattice is complete.

(B) Sufficiency.

We only have to verify that the forgetful functor $U: K^{\top} \rightarrow K$ preserves regular cointersections. Then the situation with cogeneration is analogous to the situation with generation of subalgebras: given a $T$-algebra $(A, d)$ and a regular quotient $e: A \rightarrow Y$, the cointersection $\varepsilon$ of all quotient algebras, contained in $e$, is a quotient algebra. Evidently, $\varepsilon$ is cogenerated by $e$.

By Theorem IV.2, $T$ preserves colimits of sequences of split monics. It follows from Corollary III. 4 that $U$ preserves finite cointersections. It also preserves (indeed, creates) well-ordered cointersections, since $T$ 
preserves colimits of quotient chains (which are well-ordered cointersections, as mentioned in II.I). Since $K^{\top}$ is co-well powered, there follows that $U$ preserves all cointersections.

(C) Necessity.

By Theorem I.3, $T$ preserves cointersections, hence it preserves colimits of quotient chains. Let us verify that it is right exact. Given an equivalence $e^{\prime}, e^{\prime \prime}: E \rightarrow X$, via reflexivity (which is the only property we shall need) there exists $i: X \rightarrow E$ with $e^{\prime} \cdot i=e^{\prime \prime} \cdot i=1$ (this is the same as $\left.\Delta=\left(e^{\prime} \times e^{\prime \prime}\right) \cdot i\right)$. Therefore, $e^{\prime}, e^{\prime \prime}$ are regular epis and so $T$ preserves their pushout. But in this case, pushout and coequalizer are one and the same thing. More in detail; if $k$ is the coequalizer of $e^{\prime}, e^{\prime \prime}$ then

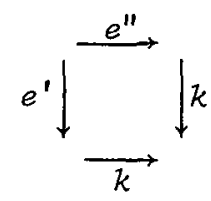

is a pushout. Indeed, given $f^{\prime}, f^{\prime \prime}$ with $f^{\prime} \cdot e^{\prime}=f^{\prime \prime} \cdot e^{\prime \prime}$, then $f^{\prime}=f^{\prime} \cdot e^{\prime} \cdot i=f^{\prime \prime} \cdot e^{\prime \prime} \cdot i=f^{\prime \prime}$; hence $f^{\prime \prime} \cdot e^{\prime}=f^{\prime \prime} \cdot e^{\prime \prime}$; therefore $f^{\prime}$ (as well as $f^{\prime \prime}$ ) factorizes through $k$.

This concludes the proof.

COROLLARY. Let $K$ be a strictly regular, co-well powered category. Then a sufficient condition for a triple $T$ to admit regular cogeneration is: $T$ is right exact and preserves colimits of chains of monics. If, moreover, $T$ preserves finite intersections and $K$ fulfils (ii) and (iii) from IV.3, then this condition is also necessary.

NOTE. Let $K$ be the category of sets or of vector spaces over a given field. Then every triple is right exact and preserves finite intersections. Therefore, cogeneration is equivalent to the preservation of colimits of quotient chains or to the preservation of well-ordered unions. This was already proved in [3]. 


\section{References}

[1] Jiři Adámek, "Automata and categories: finiteness contra minimality", Mathematical foundations of computer science 1975, 160-166 (Lecture Notes in Computer Science, 32. SpringerVerlag, Berlin, Heidelberg, New York, 1975).

[2] Jiři Adámek, "Cogeneration and minimal realization", Comment. Math. Univ. Carolinae (to appear).

[3] Jiř́i Adámek, "Realization theory for automata in categories", J. Pure App Z. Algebra (to appear).

[4] Jirí Adámek, "On the cogeneration of algebras", submitted.

[5] M.A. Arbib, E.G. Manes, "A categorist's view of automata and systems", Category theory applied to computation and control, 51-64 (Lecture Notes in Computer Science, 25. Springer-Verlag, Berlin, Heidelberg, New York, 1975).

[6] Michael Barr, "Exact categories", Exact categories and categories of sheaves, 1-120 (Lecture Notes in Mathematics, 236. SpringerVerlag, Berlin, Heidelberg, New York, 1971).

[7] Michael Barr, "Right exact functors", J. Pure Appl. Algebra 4 (1974), $1-8$.

[8] Pierre Antoine Grillet, "Regular categories", Exact categories and categories of sheaves, 121-222 (Lecture Notes in Mathematics, 236. Springer-Verlag, Berlin, Heidelberg, New York, 1971).

[9] Zdeněk Hedrlín and Aleš Pultr, "On categorical embeddings of topological structures into algebraic", Comment. Math. Univ. Carolinae 7 (1966), 377-400.

[10] Jan Reiterman, "A left adjoint construction related to free triples", J. Pure Appl. Algebra (to appear).

[11] Vēra Trnková, "On minimal realizations of behavior maps in categorial automata theory", Comment. Math. Univ. Carolinae 15 (1974), 555-566.

Faculty of Electrical Engineering,

Ceské Vysoké Učení Technické v Praze, Czechoslovakia. 